\title{
CAUCHY SEQUENCES IN SEMIMETRIC SPACES
}

DENNIS K. BURKE

\begin{abstract}
As the main result we prove that every semimetrizable space has a semimetric for which every convergent sequence has a Cauchy subsequence. This result is used to show that a $T_{1}$ space $X$ is semimetrizable if and only if it is a pseudo-open $\pi$-image of a metric space.
\end{abstract}

In a metric space it is easy to show that every convergent sequence is a Cauchy sequence; however, there are numerous examples of semimetric spaces in which there exist convergent sequences with no Cauchy subsequence. The main purpose of this note is to show that every semimetrizable space has a compatible semimetric for which every convergent sequence has a Cauchy subsequence.

Unless otherwise stated no separation axioms are assumed, and we let $N$ denote the set of natural numbers.

Let $X$ be a topological space and $d$ a real valued, nonnegative symmetric function defined on $X \times X$ such that $a(x, y)=0$ if and only if $x=y$. The function $d$ is called a semimetric for $X$ provided: For $A \subseteq X, x \in \bar{A}$ if and only if $d(x, A)=\inf \{d(x, a): a \in A\}=0$. The function $d$ is calied a symmetric [3] for $X$ provided: A set $A \subseteq X$ is closed if and orily if $d(x, A)>0$ for any $x \in X-A$. See [4] for a discussion of the differences between a semimetric space and a symmetric space.

If $(X, d)$ is a semimetric space a sequence $\left\{x_{n}\right\}_{n=1}^{\infty}$ in $X$ is said to be Cauchy if for any $\varepsilon>0$ there is some $k \in N$ such that $d\left(x_{n}, x_{m}\right)<\varepsilon$ for all $n, m \geqq k$. See [8] for the definition of weak completeness in semimetric spaces which can be stated in terms of Cauchy sequences.

EXAMPLE. Let $X=A \cup B$ where $A=\left\{(0, y) \subseteq R^{2}:-1 \leqq y \leqq 1\right\}$ and $B$ is the graph of the equation $y=\sin (1 / x)$ for $0<x \leqq 1$. If $u=\left(x_{1}, y_{1}\right)$ and $v=$ $\left(x_{2}, y_{2}\right)$ are elements of $X$ define $d(u, v)=\left[\left(x_{1}-x_{2}\right)^{2}+\left(y_{1}-y_{2}\right)^{2}\right]^{1 / 2}$ if at least one of $u$ or $v$ is in $A$. Define $d(u, v)$ to be the "arc length" between $u$ and $v$ if both $u$ and $v$ are in $B$. Then $d$ is a semimetric for the usual topology on $X$ and there are no Cauchy sequences in $B$ which converge to points in $A$.

Presented to the Society, January 23, 1971 ; received by the editors April 19, 1971.

AMS 1970 subject classifications. Primary 54E25; Secondary 54E30.

Key nords and phrases. Semimetric space, symmetric space, Cauchy sequence, weak condition of Cauchy, developable space, pseudo open map, $\pi$-map. 
THEOREM 1. If $(X, d)$ is any semimetric space (or symmetric space) the following conditions are equivalent:

(a) Every convergent sequence in $X$ has a Cauchy subsequence.

(b) If $\left\{x_{n}\right\}_{n=1}^{\infty}$ is a convergent sequence in $X$ and $\varepsilon$ is a positive number there is a subsequence $\left\{z_{n}\right\}_{n=1}^{\infty}$ of $\left\{x_{n}\right\}_{n=1}^{\infty}$ such that $d\left(z_{n}, z_{m}\right)<\varepsilon$ for all $n$, $m \in N$.

(c) If $F \subseteq X$ and there is a positive $\varepsilon$ such that $d(x, y) \geqq \varepsilon$ for all distinct $x, y \in F$ then $F$ is closed.

Proof. The proofs that (b) $\Rightarrow(a)$ and $(a) \Rightarrow$ (c) are relatively easy and are left to the reader.

To show that $(\mathrm{c}) \Rightarrow(\mathrm{b})$ we assume (c) is true and let $\left\{x_{n}\right\}_{n=1}^{\infty}$ be a sequence in $X$ which converges to $x \in X$. We may assume $x \neq x_{n}$ for any $n \in N$. Let $\varepsilon>0$. Suppose that for every subsequence $\left\{y_{n}\right\}_{n=1}^{\infty}$ of $\left\{x_{n}\right\}_{n=1}^{\infty}$ there is a subsequence $\left\{z_{n}\right\}_{n=1}^{\infty}$ of $\left\{y_{n}\right\}_{n=1}^{\infty}$ such that $d\left(z_{1}, z_{n}\right) \geqq \varepsilon$ for all $n>1$. Then we could construct a subsequence $\left\{z_{n}^{\prime}\right\}_{n=1}^{\infty}$ such that $d\left(z_{n}^{\prime}, z_{m}^{\prime}\right) \geqq \varepsilon$ for all distinct $n, m \in N$. This is impossible; so there exists a subsequence $\left\{y_{n}\right\}_{n=1}^{\infty}$ of $\left\{x_{n}\right\}_{n=1}^{\infty}$ such that for every subsequence $\left\{z_{n}\right\}_{n=1}^{\infty}$ of $\left\{y_{n}\right\}_{n=1}^{\infty}$ we have $d\left(z_{1}, z_{n}\right)<\varepsilon$ for some $n>1$. It follows that we can find a subsequence $\left\{z_{n}^{\prime}\right\}_{n=1}^{\infty}$ of $\left\{y_{r}\right\}_{n=1}^{\infty}$ such that $d\left(z_{n}^{\prime}, z_{m}^{\prime}\right)<\varepsilon$ for all $n, m \in N$. That completes the proof of the theorem.

Theorem 1 is useful in that it illustrates the effect that condition (a) has on the topology on $X$ as in condition (c). In Theorem 2 it turns out that condition (c) is much easier to use than condition (a). Condition (c) has been discussed previously in several papers (see [2], [7], [9]), and is known as the weak condition of Cauchy. In [7] there is an example of a symmetric space which has no symmetric satisfying the weak condition of Cauchy. Hence the analogue of Theorem 2 for symmetric spaces is not true.

If $d$ is a semimetric for $X$ and $x \in X$ we let $S_{d}(x, \varepsilon)=\{y \in X: d(x, y)<\varepsilon\}$ denote the sphere about $x$ of radius $\varepsilon$. It is easy to show that if $d$ is a semimetric for which the spheres are open sets then every convergent sequence in $X$ has a Cauchy subsequence. In [6] however, Heath has given an example of a regular semimetric space for which there is no semimetric with respect to which all spheres are open.

To further illustrate that Theorem 2 is about as strong as could be expected we point out that it is known (see [5]) that a $T_{1}$ space $X$ is developable if and only if $X$ is semimetrizable by a semimetric under which all convergent sequences are Cauchy.

THEOREM 2. Every semimetric space $(X, d)$ is semimetrizable by a compatible semimetric $\rho$ where every convergent sequence in $X$ has a Cauchy subsequence. 
Proof. For any nonnegative real number $r$ we define

$$
\mathscr{B}(r)=\{B \subseteq X: d(x, y) \geqq r \text { for all } x, y \in B, x \neq y\} .
$$

Let $x, y$ be arbitrary elements of $X$ and define

$$
A(x, y)=\{z: \text { there exists } B \in \mathscr{B}(d(x, y) / 2) \text {, with } z \in \bar{B} \text { and } x, y \in B\} \text {. }
$$

Now define $\rho(x, y)=\inf \{d(x, z)+d(z, y): z \in A(x, y)\}$.

It is clear that $\rho(x, y)=\rho(y, x)$ and $\rho(x, y) \geqq 0$ for any $x, y \in X$. We also note that $\rho(x, y) \leqq d(x, y)$, so

$$
S_{d}(x, \varepsilon)=\{y \in X: d(x, y)<\varepsilon\} \subseteq S_{\rho}(x, \varepsilon)=\{y \in X: \rho(x, y)<\varepsilon\}
$$

for $x \in X$ and $\varepsilon>0$.

Next we show that for $x \in X$ and $\varepsilon>0$ there exists an integer $m$ such that $S_{\rho}(x, 1 / m) \subseteq S_{d}(x, \varepsilon)$. From this it follows that $\rho(x, y)>0$ if $x \neq y$ and $\rho$ is a semimetric for the topology induced by $d$.

Assume otherwise and suppose there exists $y_{n} \in S_{\rho}(x, 1 / n)-S_{d}(x, \varepsilon)$ for each $n \in N$. Consider an arbitrary $n \in N$ such that $1 / n<\varepsilon$. Then $d\left(x, y_{n}\right)=$ $\varepsilon_{n} \geqq \varepsilon$ and $\rho\left(x, y_{n}\right)<1 / n<\varepsilon$ implies that there is a $z_{n} \in A\left(x, y_{n}\right)$ such that $d\left(x, z_{n}\right)+d\left(z_{n}, y_{n}\right)<1 / n$. Hence $d\left(x, z_{n}\right)<1 / n$. Since $z_{n} \in A\left(x, y_{n}\right)$, there is $B_{n} \in \mathscr{B}\left(\varepsilon_{n} / 2\right)$ such that $x, y_{n} \in B_{n}$ and $z_{n} \in \bar{B}_{n}$. Let $\left\{z_{n, i}\right\}_{i=1}^{\infty}$ be a sequence in $B_{n}$ such that $z_{n, i} \rightarrow z_{n}$. Now $d\left(x, z_{n}\right)<1 / n$, so $z_{n} \rightarrow x$ and there exists $m \in N$ such that $1 / m<\varepsilon / 2$ and $z_{m} \in \operatorname{int}\left(S_{d}(x, \varepsilon / 2)\right)$. Hence int $\left(S_{d}(x, \varepsilon / 2)\right)$ is an open set about $z_{m}$ and there must exist $i \in N$ such that $z_{m, i} \in \operatorname{int}\left(S_{d}(x, \varepsilon / 2)\right)$. This implies $d\left(x, z_{m . i}\right)<\varepsilon / 2$. But $z_{m, i} \in B_{m} \in \mathscr{B}\left(\varepsilon_{m} / 2\right)$ and $x \in B_{m}$, which implies $d\left(x, z_{m, i}\right) \geqq \varepsilon_{m} / 2$. This is a contradiction since $\varepsilon_{m} \geqq \varepsilon$. Hence there exists $n$ such that $S_{\rho}(x, 1 / n) \subseteq S_{d}(x, \varepsilon)$ and it follows that $\rho$ is a semimetric compatible with the topology on $X$.

To complete the proof of the theorem we show that $(X, \rho)$ satisfies condition (c) of Theorem 1. Suppose $F$ is a subset of $X$ and $\varepsilon$ is a positive number such that $\rho(x, y) \geqq \varepsilon$ when $x, y \in F, x \neq y$. If $F$ is not closed let $z \in F-F$ and let $B=F \cap S_{d}(z, \varepsilon / 2)$. Let $\varepsilon_{1}=\inf \{d(x, y): x, y \in B\}$; then $\varepsilon_{1} \geqq \varepsilon>0$ and $B \in \mathscr{B}\left(\varepsilon_{1}\right)$. There must be $x, y \in B$ such that $\varepsilon_{1} \leqq d(x, y)<2 \varepsilon_{1}$. Hence $d(x, y) / 2<\varepsilon_{1}$ and it follows that $B \in \mathscr{B}(d(x, y) / 2)$. Also $z \in \bar{B}$, so $z \in A(x, y)$. Hence $\rho(x, y) \leqq d(x, z)+d(z, y)<\varepsilon / 2+\varepsilon / 2=\varepsilon$. This is impossible since $x, y \in F$ and $\rho(x, y) \geqq \varepsilon$. So $F$ is closed.

It is worthwhile noting that if $(X, d)$ is a semimetric space which already satisfies condition (a) of Theorem 1 and if $\rho$ is the semimetric as constructed in the proof of Theorem 2 then $\rho=d$.

As an application of Theorem 2 we have the corollary below. See [3] for the definition of a $\pi$-mapping and a pseudo-open mapping as well as a discussion of related results on characterizing certain topological spaces as images of metric spaces. 
Corollary 3. A $T_{1}$ space $X$ is semimetrizable if and only if it is a pseudo-open $\pi$-image of a metric space.

Proof. Alexander [1] has shown that a $T_{1}$ space $X$ is a pseudo-open $\pi$-image of a metric space if and only if it is semimetrizable with a semimetric satisfying (a) of Theorem 1. Apply Theorem 2 and the proof is complete.

\section{REFERENCES}

1. Charles C. Alexander, Semi-developable spaces and quotient images of metric spaces, Pacific J. Math. 37 (1971), 277-293.

2. A. V. Arhangel'skii, Behavior of metrizability under factor mappings, Dokl. Akad. Nauk SSSR 164 (1965), 247-250=Soviet Math. Dokl. 6 (1965), 1187-1190. MR 33 \#697.

3. - Mappings and spaces, Uspehi Mat. Nauk 21 (1966), no. 4 (130), 133-184= Russian Math. Surveys 21 (1966), no. 4, 115-162. MR 37 \#3534.

4. H. R. Bennett and M. H. Hall, Semi-metric functions and semi-metric spaces, Proc. Washington State Univ. Conf. on General Topology (Pullman, Wash., 1970), Pi Mu Epsilon, Dept. of Math., Washington State University, Pullman, Wash., 1970, pp. 34-38. MR 42 \#2426.

5. Morton Brown, Semi-metric spaces, Summer Institute on Set Theoretic Topology, Madison, Wisconsin, Amer. Math. Soc., Providence, R.I., 1955, pp. 62-64.

6. R. W. Heath, A regular semi-metric space for which there is no semi-metric under which all spheres are open, Proc. Amer. Math. Soc. 12 (1961), 810-811. MR 23 \#A2862.

7. J. A. Kofner, On a new class of spaces and some problems of symmetrizable theory, Dokl. Akad. Nauk SSSR 187 (1969), 270-273=Soviet Math. Dokl. 10 (1969), 845-848. MR 40 \#1964.

8. L. F. McAuley, A relation between perfect separability, completeness, and normality in semi-metric spaces, Pacific J. Math. 6 (1956), 315-326. MR 18, 325.

9. S. Nedev, Continuous and semicontinuous o-metrics, Dokl. Akad. Nauk SSSR 193 (1970), 531-534=Soviet Math. Dokl. 11 (1970), 975-978.

Department of Mathematics, Miami University, OXford, OHIO 45056 